\title{
Lapurdum
}

LAPURDUM Euskal ikerketen aldizkaria | Revue d'études basques |

Revista de estudios vascos | Basque studies review

$5 \mid 2000$

Numéro $\mathrm{V}$

\section{Goulven Pennaod, Jon Mirande et la Bretagne}

\section{Francis Favereau}

\section{OpenEdition \\ Journals}

Édition électronique

URL : http://journals.openedition.org/lapurdum/1324

DOI : $10.4000 /$ lapurdum. 1324

ISSN : 1965-0655

Éditeur

IKER

Édition imprimée

Date de publication : 1 octobre 2000

Pagination : 293-305

ISBN : 2-84127-161-7

ISSN : $1273-3830$

\section{Référence électronique}

Francis Favereau, « Goulven Pennaod, Jon Mirande et la Bretagne », Lapurdum [En ligne], 5 | 2000, mis en ligne le 01 juin 2009, consulté le 03 juillet 2020. URL : http://journals.openedition.org/lapurdum/ 1324 ; DOI : https://doi.org/10.4000/lapurdum.1324 


\title{
Goulven Pennaod, Jon Mirande et la Bretagne
}

\author{
Francis Favereau \\ Laboratoire Bretagne et pays celtiques \\ Université de Rennes 2
}

De son vrai nom Georges Pinault (Saint-Malo 1928-), "Goulven Pennaod " (c'est son nom bretonnisé, comme c'était alors l'habitude militante, plutôt qu'un nom de plume) apprend le breton assez jeune, après des études secondaires au collège de Saint-Malo, où ses parents tenaient commerce, a-t-il rappelé récemment. Abeozen $(1957,216)$ le décrit arrivant, encore adolescent, dans sa cellule à la fin 1944, alors que lui-même avait été arrêté et emprisonné pour ses activités bretonnantes, notamment sur les ondes de Radio-Roazhon, la radio en breton créée sous l'Occupation (elle émettait des émissions essentiellement culturelles, mais sous autorité allemande, dirigées par L.P. Nemo, alias Roparz Hemon).

De fait, selon son témoignage postérieur ( $A L L I A M M \mathrm{n}^{\circ}$ 294/76), G. Pinault fut condamné à l'indignité nationale après la Libération, avec interdiction de résider dans les cinq départements bretons, comme tant d'autres, mais étant mineur, il fut autorisé à devenir pensionnaire dans un lycée rennais. Il y reprit donc ses études, comme le note Abeozen, après l'été 1945 où il avait été libéré. Mais il n'était pas homme à se ranger et il partit travailler à Paris dans une banque. Après un an passé au Pays de Galles (où il fit toutes sortes de métiers, tour à tour vacher, employé d'un prêtre catholique..., avant de rentrer, fuyant précipitamment devant Scotland Yard sous l'accusation de «terrorisme », F. Elies dixit, sans doute selon ses dires), il effectue son service militaire, puis il s'engage dans l'armée, et y reste faire carrière, notamment en Indochine. Il a ainsi participé à la bataille de DienBien-Phu, puis à d'autres campagnes, pour terminer sa carrière comme officier supérieur à Paris, oủ il s'est depuis longtemps installé, avant de revenir plus souvent à Saint-Malo à l'heure de la retraite.

Il a été influencé par Kerverzhioù', aussi bien dans sa poésie (parue dans les premiers numéros de la revue $A L$ LIAMM) qu'au travers de ses idées « non-chrétiennes » (Abeozen 1957, 216 - cf. nº 12-13, \& 14, 27, 32-33, 43 - traduit du suédois -, \& n ${ }^{\circ} 53$ : Kentel-zigeriñ evit ar bloaz nevez). Puis vinrent des textes en prose consacrés à l'Indochine ( $\operatorname{a} a m N g o ̂ c ~ S y, n^{\circ} 42$ ), ou à la bataille perdue de DienBien-Phu (Diskenn a eure d'an Infernioù, $\mathrm{n}^{\circ} 50$ ), alors qu'engagé dans l'armée coloniale française après la dernière guerre, où il avait été un jeune militant de 
Breiz Atao (mouvement autonomiste, c'est pour cela qu'il fut emprisonné en 1944), il côtoyait en Indochine des officiers comme J. M. Le Pen, avec qui il conservera des relations amicales de militaire engagé partageant les mêmes valeurs ; il appellera publiquement à voter pour lui dans les années 80 , malgré son « réformisme 》(sic).

C'est que, de notoriété publique, Pennaod a gardé des sympathies qui vont à l'extrême de la droite française, et plutôt dans les cercles néo-nazis. Le grand homme des revues éphémères et confidentielles qu'il a parfois fondées, comme AR STOURMER, puis DIASPAD... (cf. Dyaspat, AL LIAMM, $\mathrm{n}^{\circ} 70$ - le gallois diasbad est traduit par « cri »), ou auxquelles il a pu collaborer, comme jadis à la feuille raciste $A N N E R Z H$ (« La Force »- avec des traductions -, cf. AL LIAMM, $\mathrm{n}^{\circ} 169$ ) ou KANNADIG KER VREIZH... semble bien être Mordrel, ancien leader de Breiz Atao depuis les années vingt, qui tenta de négocier avec les Allemands en 1940, puis se réfugia en Argentine à la Libération, avant de revenir, via l'Espagne de Franco où il vécut un temps, dans les années soixante-dix.

Abeozen, le meilleur analyste de la littérature de cette période (op. cit.) ne cachait pas une certaine sympathie pour ce néo-bretonnant atypique, qui « avec ce qu'il sait a forgé un outil de discours qui nous laisse bien perplexe ", mais il avouait n'avoir « rien compris » à ces premiers poèmes de Pennaod, avant de s'y habituer, dit-il, même si son jugement global restait nuancé sur cette variante de breton jugée « élitiste ».

La période de l'après-guerre, parfois décrite comme une « traversée du désert » par la mouvance bretonne jusqu'en 1968, voit alors la traduction par G. Pennaod de certains textes de Jon Mirande, poète basque (souletin), né à Paris en 1925 (mort en 1972), travaillant au ministère de la Guerre et partageant les mêmes idées dites « extrêmes » («fascistes », disent clairement les Basques du sud, comme du nord, qui le considèrent cependant comme un grand poète et prosateur de langue basque, y compris dans les manuels scolaires récents : "faxista eta anti-semita, ez kristau, ez sozialista, tabu guztiak, baita sexuala ere, hautsi nahi zituena, garaiko euskal munduan tokirik aurkitu ezinik ibili zen. - Euskal Literaturaren Antologia, 1998, p. $94 \ldots)$.

On ne peut douter de leur influence mutuelle. Un même numéro de la revue $A L$ $\operatorname{LIAMM}\left(1966, \mathrm{n}^{\circ} 116\right)$ contient sa traduction d'une conférence faite en 1963 en Biscaye (publiée par Euskera 8-9, 1963-1964), qui présente Mirande en conclusion comme « l'un des plus importants des lettres basques de ces dernières années 》).

Dans le texte breton, intitulé "Ur barzh euskarek a-vremañ " ("Un poète basque contemporain »), qui retrace l'itinéraire de Jon Mirande Aiphasorro, on apprenait qu'il pratique, parmi de nombreuses autres, nos langues celtiques, " c'est-à-dire le breton, le gallois et l'irlandais » et qu'il a publié dans plusieurs revues, " dont en breton dans celle appelée $A R S T O U R M E R$, qu'il a même co-dirigée avec $G$. Pennaod ». Vient ensuite une traduction en breton du bref poème Igelak, puis du début de Zergatik, avec le texte basque correspondant, mais en breton seulement, de quelques strophes (première, troisième et dernière strophes) de Lili bat, puis des premiers vers de Neskatxak. 
Les relations entre Goulven Pennaod et Jon Mirande se sont nouées dès l'aprèsguerre par l'intermédiaire de Ker Vreizh, cercle de Bretons de Paris déjà évoqué. Il y a, sans doute, appris le breton auprès de ses amis, d'ailleurs, nous semble-t-il, avant de connaître Pennaod; mais il a aussi participé à divers camps bretonnants, comme $K E A V$ ( Camp Interceltique des bretonnants »), au tournant des années cinquante et soixante. On trouve d'ailleurs des échos de son passage dans diverses notes de la revue $A L L I A M M$, dont certains responsables ont bien connu Jon Mirande, semble-t-il, surtout lors de leurs séjours en région parisienne.

P. Le Bihan, le poète Per Diolier, en parle comme d'un compagnon de Kêr$V$ reizh dès l'immédiat après-guerre, dans son témoignage récent paru à l'occasion de la disparition d'André Latimier (un des cofondateurs de la revue), où il explique comment, à partit de 1942, ils fréquentaient «Kêr-Vreizh, le foyer breton, rue Sainte-Placide... et les bureaux du P.N.B. tout près, rue de Rennes. Le samedi soir se rassemblaient à Kêr-Vreizh pas mal de gens, jeunes ou plus âgés, pour discuter, emprunter des livres, assister à des conférences... ». Et de citer Jon Mirande, parmi des personnalités qui ont eu depuis des parcours très divers $\left(A L L I A M M, \mathrm{n}^{\circ} 310\right.$, 1998 , p. $400 \ldots$..). Une note précédente nous indique, par ailleurs, que Mirande a participé à une série de conférences organisée par «Kêr-Vreiz» en 1967, y parlant en breton le 18 novembre du Pays Basque (« son peuple, sa langue, sa littérature ») ou, ici en breton, Euskadi : ar bobl, ar yezh, al lennegezh (AL LIAMM, $\mathrm{n}^{\circ} 124$, 1967, p. 454). Une autre note plus ancienne témoigne également de cette intimité :

« Nous avons également appris la mort, en mars, de la mère de notre collègue, abonné de la revue, Jon Mirande. M. Mirande, qui est Basque, a appris le breton, l'écrit, ou le parle très bien ».

(traduit par l'auteur, revue $A L L L A M M, \mathrm{n}^{\circ} 67,1958, \mathrm{p} .151$ ).

Voici, à partir d'un article paru dans la revue (1986), quelle fut la « la genèse de Ker Vreizh ». L'auteur (Y.B. Tillenon, l'un de ses jeunes responsables d'alors, de la même mouvance) la situe bien parmi les émigrés bretons de la capitale qui se réunissaient dans les cafés de Montparnasse et avaient fondé, en 1936, la revue $S A V$. Tous étaient des nationalistes bretons et c'est en 1938 que fut fondé ce « centre des bretonnants et patriotes bretons à Paris ». Mais, «émanation du deuxième mouvement breton », elle avait connu des avatars : elle fut fermée par Daladier en 1939 (interdiction du P.N.B.), puis après avoir réouvert sous l'Occupation, fut inquiétée à la Libération (local et bibliothèque fermés). L'essentiel fut préservé, lit-on, " grâce à la prise en main provisoire de l'association par les Bretons Emancipés, filiale du P.C.F. dont le président d'honneur était Marcel Cachin ». Après avoir été administrée par des " modérés » (P. Laurent), elle connut dans les années soixante « un tournant plus nationalitaire » sous la présidence de Kerlann (J. Delalande, ancien responsable d' $A r$ Falz). Puis, à partir de 1965, commencèrent des cours du « nouveau breton », dit moderne, tel que certains nationalistes rêvaient qu'il fût.

L'auteur ajoute : « Nous pouvions y rencontrer des Européens de toutes origines. Jon Mirande-Aiphasorro (sic) était basque - l'un des deux ou trois premiers poètes et écrivains de son pays - et aussi un des plus brillants connaisseurs du breton moderne, d'autres venaient de Frise, d'Irlande, de Flandres, de Galles ». Puis 
KerVreizh connut un déclin avant de renaître dans un esprit « celtique», voire druidique, mais ouvert à « l'avenir de l'Europe». On y enseigne alors un breton dit « moderne » (le « néo-breton 》), et le breton ancien et moyen, comme les origines « indo-européennes » (cf. numéros des revues DIASPAD, KER VREIZH... - \& cf. AL LIAMM, $\mathrm{n}^{\circ} 222,1984$, sur le numéro 4, par exemple) ; mais on y trouve surtout, au travers de ces publications, des références à la nouvelle droite, au néopaganisme etc., alors que les animateurs de ces entreprises affirment se faire souvent traiter de « fascistes ».

G. Pennaod a également évoqué Mirande dans la préface d'un manuel de basque en breton, à paraître. Il fut, en effet, dans ces années d'après-guerre, un des compagnons de celui qui est considéré aujourd'hui comme un des grands poètes basques du siècle, mais cependant présenté comme un Basque (français) aux idées ouvertement fascistes, racistes, antisémites et xénophobes, ce qui semble conforme à ses écrits. On trouve même, dans les pages de sa revue confidentielle AR STOURMER des textes d'un goût détestable, pour ne pas dire plus, comme un poème de Pennaod et Mirande qui narre le viol d'une très jeune noire.

Quant aux relations entre Jon Mirande et la Bretagne, elles font l'objet d'un témoignage personnel en breton de G. Pennaod (février 1998 - cf. plus bas). On y voit quelques individualités (Pennaod, Mirande, Jestin), d'autant plus isolées qu'elles sont " anticonformistes » et brillantes, tenter de marier leur basquisme et bretonnisme avec un néo-paganisme inspiré des mythes celtiques et indo-européens, mais surtout, dans un discours délirant, voire ordurier, tenter de concilier basquitude ou bretonnitude et élucubrations idéologiques d'aspect savant ou érudit (philologique), mais de type ouvertement néo-fasciste, antisémite, clairement pro-SS, dans une course singulière à l'originalité par rapport à la «tribu » des bardes en question...

À titre d'exemple, le ${ }^{\circ} 6$ (novembre-décembre 1962, p. 63), contient un écrit qui est une traduction d'un poème de René de Clerc, Niet iedereen kan heiden zijn ( «Tout le monde ne peut pas être païen »). Puis, sous le titre général « Notre conviction », on y lit des déclarations de «Rigakos » [Rieg Jestin-alias Raymond Gestin], G.P. [Goulven Pennaod] et J.M. [Jon Mirande] (p. 70) sur une spiritualité qui se voudrait païenne etc. D'autres numéros, nous dit l'auteur, sont trop « politiquement incorrects » aujourd'hui pour être mentionnés (en raison de nouvelles lois, probablement).

G. Pennaod publiait également, entre-temps, des notes d' « ethnolinguistique» (Notennoù a Genelyezhoniezh-AL LIAMM, $\mathrm{n}^{\circ} 95$ etc.), parfois vertement critiquées par l'abbé Le Floc'h, le poète Maodez Glanndour, lui-même grand poète mais théologien plutôt droitier et traditionaliste, ici du point de vue de la tradition judéo-chrétienne (pour le sens du mot « verbe » par exemple, au sens biblique, défendu contre les « néo-païens ») ; mais il a surtout beaucoup collaboré à la mise en place d'une terminologie «moderne » en relation étroite avec le docteur Etienne, sous l'édige du mouvement SADED (KIS-SADED, c'est-à-dire Kreizenn Imbourc'h Sturyezhouriezh - "Centre de Recherche en Linguistique normative »), à partir des années soixante, empruntant au breton ancien nouvellement réexaminé 
(par les travaux de L. Fleuriot, notamment) comme aux langues celtiques (en particulier l'irlandais dans les domaines « officiels » etc.). Le moins qu'on puisse dire est que le discours accompagnant cette création néologique, autant que l'idéologie qui la soutend, sont loin d'être neutres. Ils prônent, en tout cas, un État fort, seul capable de rebretonniser la "masse » selon leurs vues ultra-nationalistes ef très « sectaires »...

En dehors de quelques autres textes de fiction (comme la nouvelle Ar c'hloareg - « Le séminariste » - dans AL LIAMM, n 139, 1970 - où le « héros » narrateur, sans doute alter ego de l'auteur, tue un communiste participant à une contre-manifestation anti-fasciste, sur fond de lutte pro-OAS), de divers autres témoignages (cf. $\mathrm{n}^{\circ} 122,1967$ : Ich hatte einen Kameraden), il a fait paraître beaucoup de traductions, des langues les plus diverses, du poète breton de langue française, le très notable Charles Le Quintrec ( $\mathrm{n}^{\circ} 115$ \& 116, 1966), au poète « lache " Ondra Lysohorsky ( ${ }^{\circ} 123,1967$ - plusieurs poèmes traduits, parfois avec R. Huon), ou à l'espagnol (nº 126, 1968 - M.A. Asturias) etc.

G. Pennaod s'est également fait connaître par des études érudites sur plusieurs langues anciennes rares, comme sur le vieux-breton (à l'École Pratique des Hautes Etudes) et sur le gaulois (entre autres travaux par la publication, en collaboration, du calendrier de Coligny, aux éditions CNRS, vers 1980) ; un moment chargé de: cours de gaulois à Lyon III, il en a été écarté à la fin des années quatre-vingt, lors de l'exclusion par le ministre (Jospin) d'enseignants jugés " révisionnistes » (Notin) ou néo-nazis dans cette même université (d'après le journal Libération).

Il a publié un manuel de moyen-breton, Dornlevr krennvrezhonek (PREDER $\mathrm{n}^{\circ}$ 59-61, 1964), ainsi que des « notes » linguistiques, tant dans HOR YEZH (cf. $\mathrm{n}^{\circ} 53-54,1964$ ) que, plus tard, dans $A L \operatorname{LIAMM}\left(\mathrm{n}^{\circ} 114,142 \ldots\right.$ ). Il a ensuite publié des critiques linguistiques portant sur la réédition du Catholicon $\left(\mathrm{n}^{\circ} 136\right)$, ainsi que des grammaires de Trépos $\left(\mathrm{n}^{\circ} 130\right)$, Hemon en anglais $\left(\mathrm{n}^{\circ} 171\right)$, de manuels de breton ( $\left.\mathrm{n}^{\circ} 199\right)$, puis plus récemment d'un ouvrage sur la langue gauloise ( ${ }^{\circ} 287,1994$ - Un deskrivadur a'r galianeg), sur le yiddisch $\left(\mathrm{n}^{\circ} 295\right)$, la néologie... chez les autres ( $\mathrm{n}^{\circ} 304-$ Foerell ar gerioù nevez), ainsi que notre propre grammaire du breton contemporain ( ${ }^{\circ} 306,1997$ ), au point, semble-t-il, d'être devenu le critique attitré de cette revue, au moins dans le domaine des études linguistiques, ce qui ne laisse pas d'étonner. Il a également écrit, nous a-t-il confié à Saint-Malo en 1996 (après avoir assisté à notre conférence sur P. J. Hélias) un projet de grammaire « supradialectale » et un manuel de basque en breton, à paraître.

Il a été critique, notamment littéraire, pour LA BRETAGNE PARIS (son pseudonyme est Fanch Trimer), dont les critiques n'ont pas toujours fait l'unanimité (cf. YB Piriou 1978, LE PEUPLE BRETON, n 171, à propos de son jugement sur la poésie du directeur de la revue $A L L I A M M$, Ronan Huon, qualifiée de «méchants poèmes ", par celui qui se vantait plutôt d'être un «anti-poète 》).

Un article sur le théâtre breton paru en 1995 chez Bordas-Larousse (Dictionnaire encyclopédie du théâtre), partial à souhait et le plus souvent mal informé, nous éclaire sur son analyse, qui est tout à fait perverse : « en tant que 
langue, le breton est mort puisqu'il n'existe pas plus de dix monolingues probablement nonagénaires » (p. 495), même s'il se trouve « une élite intellectuelle de mainteneurs " ... Nous pensons qu'il confond là monolingues et bretonnants, aujourd'hui nécessairement bilingues et métissés ! Que ne dirait-il donc des autres, dont les Basques ? Cet article a suscité récemment la réprobation des artistes et intellectuels bretonnants qui en ont eu connaissance, notamment les membres de la troupe de théâtre bretonnant professionnelle, Strollad ar Vro Bagan, dirigée par G. Kervella, dont le propos et la pratique se situent à l'exact opposé de ces affirmations.

Sans doute y a-t-il beaucoup de provocation chez ce "personnage " à la solide réputation de baroudeur. Mais n'y a-t-il pas aussi comme une nostalgie durable pour un " ordre nouveau » apparu à l'ombre du III' Reich ?

Aussi bien, pour limitée que soit aujourd'hui une telle "sensibilité », qui se revendique d'ailleurs comme brutale (avec le culte ridicule de la « force», surtout dans leur cas !), et qu'ignore la très grande majorité des Bretons, des bretonnants et même des plus jeunes, imperméables à ces idées, en général, voire incrédules quant à leur existence, elle n'est pas totalement isolée, solidarité ancienne et vieil attachement ou compagnonnage de temps difficiles aidant, dans un « mouvement breton traditionnel » qui a pourtant globalement suivi le net virage à gauche pris depuis 1968, avant de pénétrer désormais l'ensemble de la société civile bretonne, y compris donc dans ses marges les plus extrêmes (ce " quarteron " de trois ou quatre « bretonnants » représente ainsi moins de $2 \%$ de nos 200 auteurs).

C'est dans cet esprit que nous reproduisons le témoignage de G. Pennaod sur Jon Mirande, que nous avions sollicité fin 1997 au nom de notre laboratoire de recherche (Rennes 2), à la demande de nos collègues basques (traduit du breton par nous-mêmes).

\section{Bibliographie}

ABEOZEN (Elies F.) 1957. Istor lennegezh vrezhonek an amzer-vremañ, Al Liamm (251 p.)

ELIZEGI X.M. \& IZAGIRRE K. 1998. Euskal Literafuraren Antologia, p. 94-97, Donostia.

FAVEREAU F. 1991. Littérature et écrivains bretonnants depuis 1945, Skol Vreizh (86 p.)

OLIER Y. 1968... O sellout war va c 'hiz, Imbourc 'h (plusieurs tomes de souvenirs à partir de 1940)

OLIER Y. 1974-1975. Istor hol lennegezh : skol WALARN, 2 tomes, Imbourc ' $h n^{\circ} 58 \& 61$.

PENNAOD G. 1998. Témoignage personnel de l'auteur (à ma demande, ès-qualités) : «J. Mirande ha Breiz $h$ ».

RaOUL L. 1992. Geriadur ar skrivagnerien ha yezhourien, Al liamm (434 p.)

TILLENON Y.B. 1986. "La genèse de Ker Vreizh », Kêr Vreizh, n 56. 


\section{G. PENNAOD}

\section{Jon Mirande et la Bretagne}

Con Jon Mirande, suletino y parisiense, tan poco ortodoxo commo su paisano Chaho, estamos y a muy lejos de aquellos certámenes de fines de siglo en los cuales, segrin se quejaba Unamuno, «tanto el poeta como el cuadro han de estar en armonia (sin h) con nuestra salvadora doctrina y con las... tradiciones venerandas de nuestros mayores '(porque si no, no hay arte, ni cosa que lo valga) ". Mirande, traductor de Poe y Kafka, ha dado ya, a pesar de sujuventud, abundantes pruebas de su inquietud y de su falta de respeto para con los convencionalismos, al tiempo que se ha acreditado como poeta de gusto seguro y cultivado y como versificador de consumada habilidad.

Voilà ce qu'écrivait, avant 1960 , le plus brillant érudit de notre époque en matière de basque, le Prof. Luis Michelena, disparu, hélas, il y a douze ans, à propos d'un homme jeune encore, né à Paris le 10 novembre 1925. Celui-ci est aujourd'hui considéré comme un maître par plusieurs de ses compatriotes, qu'ils soient du nord ou du sud d'Euskal herria. Mais à sa mort, vers la Noël de 1972, à peine trouvait-on depuis environ six mois une poignée de gens de son pays pour lui écrire, alors qu'il était déjà très malade. Cela lui fut d'un grand réconfort, évidemment, lors de ses derniers moments, mais il serait grandement étonné si on lui disait que l'on mettrait son œuvre au programme d'un DEA à Bayonne (et dans d'autres universités basques probablement). Grâce à la fidélité indéfectible de Txomin Peillen, ami de toujours, il commença à se faire connaître sérieusement au moment de la publication d'un choix de ses écrits [1976], qui contient au chapitre 7, Jon Mirande keltikalari, soit « JM celtisant » pp. 201-299, la preuve de l'intérêt que portait JM au monde celte [sans compter aux pages 272-281 l'article en sa mémoire, traduit par Txomin Peillen]. Il connaissait parfaitement l'irlandais, le gallois, le cornique et surtout le breton.

Etant, depuis l'adolescence, un nationaliste basque, et euskalduna de naissance pour ainsi dire, bien que né à Paris, mais de parents venant de Sohuta en Soule, il commença très tôt à avoir des relations avec d'autres nationalistes de France qui s'opposaient au jacobinisme de cet État-nation ${ }^{2}$ et durant la guerre, en 1943 me semble-t-il, il lui arriva d'aller le sāmedi après-midi à Ker Vreiz ${ }^{3}$, où il commença à apprendre le breton. Quoi qu'il en soit, il était devenu très expert lorsque je le rencontrai pour la première fois en 1947 [cf. 1960, p. 272-273]. Depuis cette période, il a échangé de nombreuses lettres dans notre langue avec Kerlann ${ }^{4}, \mathrm{Klerg}^{5}$, Hemon $^{6}$, Etienne ${ }^{7}$ et plusieurs autres ; quant à moi,j' ai reçu de lui nombre de lettres et de cartes postales des pays qu'il visitait ; toutes étaient en breton (avec des citations drôles en allemand, en anglais ou dans d'autres langues), sauf une fois où il m'écrivit une longue missive en cornique, alors que j'étais au Viet-nam, ce qui donna bien des soucis aux hommes de la Sécurité militaire... Il fallut attendre 1962, en revanche, pour voir apparaître ses premiers articles originaux en breton.

Il fut le cofondateur avec moi-même et « Rieg Jestin »", le célèbre spécialiste des Sumériens - du journal Ar Stourmer et à partir du premier numéro (janvierfévrier 1962, p. 8-9), on y trouve un article écrit de sa main, ER DEVOBIS ANMARVOLOBIS... (étonnant néo-vieux-celtique !) qui prétend « défendre nos 
races européennes », contre le fascisme « qui reste avant tout une méthode, car une attitude de sujétion à l'état, quel qu'il soit,... le plus froid des monstres froids, selon Nietzche, ne saurait vous convenir ». Il concluait ainsi : " Et quand viendra le moment du nouveau combat, que cela soit pour la gloire et l'honneur de Dazoue [du Dagda], Wodan, Ortzi et tous les dieux de nos tribus - autant de... diables', affirma le sale Juif ${ }^{10}$ - où l'on sacrifiera une fois encore dans leurs brasiers les races impures qui tentèrent de subjuguer l'univers sacré ! » Eh oui, on pouvait effectivement s'exprimer en toute franchise à cette époque !

Dans le $n^{\circ} 2$ (mars-avril 1962, p. 19), un autre article intitulé « Nouveaux dogmes » attaquait de façon virulente l'Église de Rome et les « sionistes français » à propos du « problèmes de la mono- ou poly- [?]genèse [ou - ?- mono-/polythéisme ${ }^{11}$, qui est un problème d'anthropologie pure ». Il y joignait (p. 22) une chanson, écrite «par le barde gothique illustre Horila Arsgudja » [cf. note 6 ; sens de « petit putassier du Prêtre-au-cul » ou «d'enculé de Prêtre »- ?-], soi-disant, et 《 traduit du gothique par RIGOVIX», dont le titre en gothique aurait été Hvan aftraqimand thai SS-wairos ("Quand les SS seront de retour »), ajoutant que « les vilains enfants pourront la chanter sur l'air de Julien Cadoudal ». Je ne jurerais pas qu'il s'agisse d'un chef-d'œuvre !

Dans le $n^{\circ} 4 / 5$ (septembre-octobre 1962, p. 51-52), il donne « Un regard sur l'Espagne de Franco », où il écrit : «Je suis Basque ; on m'accusera peut-être de me montrer partial envers l'Espagne et indifférent à sa grandeur. C'est exact, je ne suis pas tenté d'être trop bienveillant envers des personnages comme ce caudillo très libéral qui accorda, durant sa... croisade libératrice et au nom de notre sainte mère l'Église, Jésus-Christ notre sauveur, sa mère sacrée et tous les saints, sans doute, d'offrir à chacun de ses soldats maures un fille basque comme esclave. [...] Je regrette d'avoir vu mes compatriotes devenir, pour des raisons tactiques, les supplétifs des Rouges qui leur avaient offert une piètre autonomie quand survint la guerre intérieure, alors qu'il eût bien mieux valu lutter contre tout Espagnol pour la liberté d'Euskadi. Mais je comprends ceux qui se battaient aux côtés des hommes de gauche, toute sanguinaire que fût leur guerre, et je leur accorde des circonstances très atténuantes puisqu'ils prédisaient bien que la croisade des libérateurs se ferait au profit des évêques, des colonels et des bourgeois voraces dont on mesure à présent l'outrecuidance ».

Dans le $\mathrm{n}^{\circ} 6$ (novembre-décembre 1962, p. 63), un écrit qui est une traduction d'un poème de René de Clerc, Niet iedereen kan heiden zijn (« Tout le monde ne peut pas être paien »). Sous le titre général « Notre conviction», on y lit des déclarations de Rigakos [alias Rieg Jestin - Raymond Gestin], G.P. [Goulven Pennaod] et J.M. (p. 70) que je reproduis ici en totalité :

Je crois à l'âme - pour lui donner un nom commun - donc je suis convaincu qu'il existe dans chaque être une qualité psychologique et non pas ce $\psi$ ù $\chi \eta$, comme le prétendent les tenants d'un matérialisme mécanique, simple épiphénomène de la matière (du cerveau pour l'être humain et les autres animaux), mais, au contraire, qu'elle est distincte, dans sa manière d'être la plus profonde, des phénomènes chimiques et physiologiques communs (à mon avis, cela a été démontré, en dernière analyse, par la recherche parapsychique), si bien que l'on pour- 
rait dire que l'âme, au sens strict, est la seule réalité. Oui, j'irais jusqu'à affirmer que je suis parfois tenté de croire que mon âme à moi est la seule réalité...

À l'inverse de ce qu'enseignent certains systèmes métaphysiques (ceux de l'Inde, par exemple), je crois, quant à moi, que cette âme est personnelle, du moins d'une certaine façon, car je ne nie pas qu'elle ait également une forme interpsychique, mais, à l'inverse de l'enseignement du christianisme, je crois qu'elle préexiste à notre naissance et qu'elle est de nature active - comme elle continuera d'exister après notre mort ici-bas. Je ne veux pas signifier, cependant, que je crois en la métempsycose, c'est-à-dire à la nécessité pour toute âme de transiter successivement par un grand nombre de corps terrestres, chaque réincarnation étant dépendante de nos mérites moraux dans une vie antérieure (enseignement du Karma). Moi, je crois plutôt - comme l'avaient peut-être saisi les anciens Druides celtes - que notre âme personnelle vit quantité de vies dans des mondes dont la matérialité est différente, sur différents plans de la réalité, et peut-être, si le temps n'existe pas en soi, ces vies coexistentelles, mais c'est là un autre problème...

Mais sur tous les plans, dans chacun de ces « mondes », il faut que l'âme ait un corps matériel, parce que l'on peut dire que ce corps n'est rien d'autre, à chaque fois, que la conscience de l'âme, la façon dont celle-ci se reconnaît ( « L'âme est la forme du corps ” a dit Aristote). C'est pourquoi, bien que le corps soit chose éphémère et secondaire, si l'on veut, au regard de l'âme - destiné qu'il est à " mourir », dans la mesure où l'âme passe sur un autre plan il n'est pas méprisable, comme le pensent les gens d'esprit religieux, mais, bien au contraire, il doit être reconnu comme le fondement nécessaire de notre personnalité consciente, et partant, malgré les discours des... (die Hinterweltler), nous devons respecter ses qualités instinctives qui sont le symbole et l'emblème de l'Ame - même les plus humbles et les plus modestes, mais tout d'abord les plus profondes et importantes : je veux dire la science du sexe (gallois rhyw...) et la science de la Race. Nier la valeur de ces sciences sous prétexte de la supériorité de l'« esprit » ne serait que traîtrise et sottise, car « quiconque veut faire l'ange fait la bête ».

Parce que je crois à l'âme, devrai-je croire en Dieu ? C'est un autre problème, entièrement distinct à mon sens. Pour moi, l'âme se suffit à elle-même. Cependant, lorsque le mal de l'unité pèse sur elle, elle a alors tendance à honorer les " dieux », qu'il s'agisse seulement du fruit d'une nostalgie ou du souvenir en forme d'écho des héros de la tribu, qui continuent d'exister sur d'autres plan de l'Être.

Paris, 25/12/1962

Le style de cette déclaration est assez maladroit ; mais il ne faut pas oublier l'indigence du vocabulaire intellectuel en breton à l'époque et la difficulté qu'il y avait à manier des concepts abstraits - « non concrets » comme l'on disait. Dans la même livraison, il traduisait (p. 72-73) un article, Edo traidore edo abertzale?, publié dans la revue de $1^{\prime}$ E.T.A., Zutik, n 6, p. 1.

Dans le $\mathrm{n}^{\circ} 7$ (janvier 1964), p. 13, « Iudaica. À propos d'un poème de Ch'aul Tchernikhovski », qui mentionne ces Israéliens, généralement des intellectuels, qui rejettent Yahweh comme dieu pour revenir aux anciennes divinités du pays, se nommant eux-mêmes Canaanéens (?), ou même les dieux de l'Olympe grecque ; «Pour lui [Ch.T.], ce sont les Dieux helléniques les véritables divinités, Apollon en premier, et le Dieu d'Israël n'en est qu'une copie, qui a été déformée et dévalorisée ensuite par les Juifs », comme le montre un poème... (" Devant la statue d'Apollon »), dont Mirande donne une traduction à partir du néo-hébreu, et dont voici les derniers vers : 
Mon Dieu, Celui des déserts mystérieux,

Dieu des féroces envahisseurs du pays de Canaan,

ils l'ont attaché avec des lanières de phylactères!

Là encore, on constate à quel point J.M. avait le désir d'essayer de retrouver les " anciens dieux », comme on le voit également dans son poème basque consacré à Ortzi ou aux " Sorcières de mon pays ".

Dans le $n^{\circ} 8$ (février 1964), nous avons signé ensemble l'éditorial «Den Schweinen wird alles Schwein! ou (en breton) «Par la boue et par les ronces/C'est le métier du laquais ", dont il serait politiquement incorrect de parler plus avant ici.

Dans le $n^{\circ} 9$ (mars 1964) on trouve " Monsieur Ben Gurion est-il nationalsocialiste ? " à propos d'un article (traduit là en breton) qu'il avait publié dans L'Arche. Et un poème à la manière des Lettristes français, composé après dîner et une beuverie en tête à tête, qui eut le don de mettre Klerg (l'abbé Marcel Le Clerc) dans une colère rouge [sic].

Dans le $\mathrm{n}^{\circ} 10$ (avril 1964, p. 54-5) on trouve une traduction du néerlandais Roeland Cavallier, « Les dieux nègres ».

Il ne semble pas avoir collaboré aux numéros suivants, consacrés surtout à des questions linguistiques bretonnes et non plus à la politique. Il faut également faire état de sa participation au petit supplément que nous avions appelé $A r c$ 'hilhog gall ( «L coq gaulois »- ou « français ») qui tournait en dérision ceux que nous surnommions alors " an tousegi $\|^{12}$ ( "les crapauds » en breton), et qui était un peu dans le genre de Yod-kerc' $h^{13}$ plus tard ou, surtout, d'Igela ( $"$ Le grenouille $\left.»\right)$, la première revue « irrespectueuse » écrite dans la sainte langue des Basques et dirigée par Jon Mirande et Txomin Peillen, ce qui valut à J.M. de se voir refuser d'entrer à Euskaltzaindia (L'académie de la langue basque), sous la pression du chanoine P. Lafitte, grammairien éminent, mais également pauvre type, qui osait me dire en juillet 1973, au cours d'un repas à Aiherra (Nafarroa Garaia) - sept mois après la mort de J.M. - que «Ah, Jon : c'était le plus grand d'entre nous tous... »

(lettre du 10/02/1998) 
1. Kerverzhioù, comme on le cite en général, est la forme bretonnisée du patronyme de Guillaume Berthou (Paimpol 1908-1951 Rennes), qui a signé de nombreux autres pseudonymes (cf. Raoul 1992, 29). Fils d'un capitaine du Goélo (région dont on trouve trace dans certains écrits en prose comme Lezardrev, bien que la langue soit souvent assez standardisée), il a été élève au lycée de Saint-Brieuc, où il a obtenu le baccalauréat ès sciences et lettres (1925-1926) et, après des études de chimie à Rennes, il est reçu ingénieur en 1929. Après avoir voyagé en Grande-Bretagne et à Saint-Pierre et Miquelon, il est ingénieur à BasseIndre en 1929, où il tombe malade, puis chimiste à la poudrerie de Saint-Chamas en Provence, avant de travailler à celle du Moulin Blanc à Brest en 1931. İnstallé à Guipavas, avec sa femme qui est pharmacienne, le couple perd sa petite fille, et il est inquiété par la police après l'attentat de 1932. Sa fernme mourra à son tour, et c'est de cette période que daterait son attirance pour l'astrologie et les sciences occultes (Olier 1968, 44). Il est ensuite employé à Marseille dans une huilerie, se remarie en 1933, avant d'être sans emploi en 1934. On le retrouve à Tréguier en 1935, directeur de l'entreprise « Caïffa », puis en 1936 à Saint-Brieuc, travaillant auprès de l'abbé Vallée qui s'occupe des artisans, puis à Paris... Il deviendra professeur de chimie et de sciences naturelles dans un collège privé de Brest en 1939. Durant l'Occupation, il sera un des proches de Roparz Hemon, secrétaire de Gwalarn, trésorier d'Arvor, el collaborateur de L'heure bretonne jusqu'en 1944 (avec la chronique «Ar yezh hag ar vro »). Puis, à la fin de la guerre, il travaillera dans une imprimerie rennaise, tout en étant en 1946 trésorier de la revue druidique $K a d$, puis rédacteur du nouvel Ogam en 1948. Malade, il meurt à 43 ans.

Kerverzhioù a été un des scientifiques bretonnants de l'équipe de Gwalarn. On lui doit ainsi un petit livre, illustré par son frère, sur les insectes, Levr an amprevaned (1943), ainsi que de nombreux articles publiés dans diverses revues durant ou après la dernière guerre, dont quelques-uns sur les mathématiques ou les sciences, parfois éditées après sa mort (cf. Raoul 1992, 30). Il a publié des écrits en prose dans $A n$ Oaled, Arvor (une dizaine de nouvelles, dont certaines inspirées de contes populaires irlandais, en 1944, ainsi que le texte de chroniques faites à la radio sur les sites de Bretagne, "Lec'hioù Breizh ", comme Landreger, qui date de 1942, ou Lezardrev...). Ajoutons-y des textes publiés par Galv, Studi hag Ober, ou dans Al Liamm (Foar Vre, ${ }^{\circ} 19$ ), ainsi qu'un roman inachevé, Ar Vag Jupiter.

Mais il a surtout été poète, inspiré par l'antiquité celtique et le druidisme, influencé par le nordisme et le paganisme, qui furent de mode durant ces années quarante. Son goût des sciences occultes a déjà été souligné. Quant à la forme, il s'agit souvent d'une écriture de type ésotérique, telle qu'elle avait pu être pratiquée par Yeats en anglais, ou, en français, par Mallarmé... Il a ainsi publié une vingtaine de poèmes dans la revue Gwalarn (icf. Abeozen 1957, 132), de 1930 à 1943 (dont un plus long que la plupart des autres, Epona, $n^{\circ}$ 158). Ceux-ci ont été réédités en un volume, Barzhonegoù (qui aurait dû s’appeler Barzhaz Kerverzhioù, préparé par son proche ami Arzel Even en 1955). Abeozen (op. cité) note que le poète gallois T. GwynnJones tenait certains de ses poèmes en grande estime, peut-être à cause de leur goûts bardiques communs. Car Kerverzhioù aimait jouer avec les idées, comme avec les mots, dont parfois les plus rares, et l'approche de sa poésie est réputée pour le moins difficile. Abeozen lui-même, pourtant lettré, poète et « gwalarniste » avoue, à regret (1957, 195), n'avoir jamais pu « s'attacher à la poésie de Kerverzhioù », au contraire de celle de R. Hemon, Y.E. Jarl, ou Maodez Glanndour : "Cela est sans doute affaire de sensibilité. Cette manière d'écrire de la poésie ne trouve pas de résonance en moi... Il y a une autre raison. La théologie, tout celtique

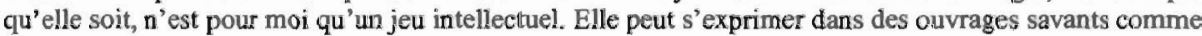
An Notennoù ou Sketla Segobrani. Cela me va. Mais je suis probablement trop marqué par le sceau du christianisme. Même s'il m'arrive d'avoir de sérieux problèmes avec la foi de mon enfance, je sais pertinemment qu'elle a fait s'élever notre peuple d'un degré dans l'humanité. La tradition ancienne ne peut être pour moi, comme pour d'autres, qu'un objet de connaissance et non de foi ». Au total, le principal reproche formulé à l'encontre de la poésie de Kerverzhioù est son cérébralisme, qui en annonce d'autres, même si on y trouve aussi une mystique de type chrétien, mariant ainsi (Abeozen op. cité), esthétique et intellect.

2. En néo-breton riez, terme reconstitué d'après le vieux-breton ried (cf. gallois rhieddog « royal "), du celtique ri, gaulois Vercingetorix, mot indo-européen apparenté au latin « rex » pour « roi », mais et surtout pour le sens à l'allemand Reich!

\section{3. cf. plus haut : " G. Pennaod, Jon Mirande et la Bretagne »...}

4. Jean Delalande (La Roche-sur-Yon 1910-1969 Morlaix), alias Kerlann, a été le successeur de Sohier (mort en 1935) à la tête d'Ar Falz. Né en Vendée, où son père était militaire, il fut élevé après la disparition de celui-ci par sa mère à Morlaix, d'où elle était originaire et où il fit ses études, avant d'aller à Brest en 1923, puis à Londres. Revenu en Bretagne, il est nommé instituteur public en 1930 (à Plomeur, Trégonc, Quimperlé, Guiclan après son service militaire en 1932 -, puis à Penmarc'h, où il reste de 1926 à 1941). C'est là, en pays bigouden et dans le Cap-Sizun (où il enseigne brièvement en 1941), qu'il recueille de nombreux proverbes, rimes, et chants populaires, «bruzun ar brezoneg ", édités en 1936 (Soniou brezonek evit 
ar skoliou), puis plus récemment par Brud Nevez. Arni de Sohier, comme de Keravel qui sera responsable d'Ar Falz après-guerre (tous deux « communisants »), il est avant la guerre une des chevilles ouvrières de la revue, dont il prend logiquement la direction à la mort de son fondateur en 1935. Mobilisé en 1939, il est fait prisonnier, avant de rentrer en Bretagne en 1941. Il devient alors secrétaire du PNB (tendance nationaliste modérée, dite Delaporte, opposé au pro-nazi et fasciste Mordrel) pour le Finistère en octobre 1942. C'est alors qu'il ouvre en novembre 1942 une école primaire libre de langue bretonne à. Plestin, la première du genre. Mais l'expérience lui coûtera cher après coup. Révoqué à la Libération, il doit s'exiler à Paris (où il côtoie d'autres «gwalarnistes » comme Abeozen...), avant de rentrer à Air-France ; c'est alors qu'il devient actif à Ker Vreiz, dont il est le président en 1966. Ses textes, chants et poèmes mais aussi ses nouvelles et articles divers, ont été publiés dans diverses revues, d'abord Gwalarn (entre 1934 et 1944), mais également Ar Falz, Breiz, Arvor, Sav... (cf. Abeozen 1957, 166), et enfin, récemment, Brud Nevez, ainsi que les différentes revues bretonnantes d'après-guerre (cf. Raoul 1992, 86).

5. L'abbé Marcel Le Clerc, alias Klerg (Plémet 1912-1984 Bégard) a été écrivain et surtout directeur de la revue catholique et bretonnante $B$ arr-heol, reprenant la tradition de Feiz ha Breiz ( ( Foi et Bretagne ») en Trégor. Ordonné en 1936, il avait été le vicaire du recteur Lec'hvien, écrivain bretonnant (auteur de livres de dévotion surtout), tué par le maquis en 1944. L'abbé Le Clerc était un ardent nationaliste (proche du FLB à la fin des années soixante), assez traditionnaliste en matière de mœurs... Il est l'auteur de nouvelles, d'éditoriaux, de traductions des poèmes du gallois ancien, de pièces de Shakespeare et de nombreux autres textes (Raoul 1992, 209).

6. Roparz Hemon est le pseudonyme de Louis Paul Nemo (Brest 1900-1978 Dublin), l'un des écrivains bretonnants les plus notables du $20^{\circ}$ siècle. Né dans une famille du Léon liée à la Marine, il fait de solides études à Brest, Paris, puis en Angleterre, et devient agrégé d'anglais à Brest en 1924. Proche de Breiz Atao, il crée en 1925 la revue Gwalarn, dont il restera l'animateur jusqu'en 1944, ainsi qu'un des principaux écrivains (traductions d'autres langues celtiques et classiques européens dans une perspective toute « moderniste $\gg$ ), en même temps que le promoteur d'initiatives culturelles à destination du public ou peuple bretonnant. Il poursuit ces tâches sous l'Occupation (Institut Celtique, Radio-Rennes), ce qui lui vaut d'être inquiété à la Libération (après qu'il a fui en Allemagne en 1944). Condamné à l'indignité nationalc, il s'exile volontairement à Dublin, où il entre à l'Institute for Advanced Studies (grâce à l'appui du président de la République d'Irlande, De Valera, dit-on). Il y publiera diverses œuvres du moyen breton et un dictionnaire historique. Vrai chef de file du mouvement nationaliste culturel, sans jamais revenir en Bretagne avant sa mort, il a édité d'Irlande le mensuel Ar Bed Keltiek (1951-1971). Auteur d'une œuvre multiforme, tant savante que littéraire (poèmes et chants, nouvelles et plusieurs romans, théâtre, traductions etc.), forte de centaines de titres, il fait figure de saint-patron de l'emsav bretonnant, révéré par d'aucuns, même si son parcours personnel et certains de ses propos lui ont valu d'être critiqué par une partie des lettrés bretonnants.

7. Guy Etienne, dit Abanna (né en 1928 à Saint-Carreuc). Psychiatre installé à Châteaulin. Connu pour avoir élevé et scolarisé ses enfants en breton dans les années 60 . Directeur du cours par correspondance, de type associatif $S A D E D$ pour le second degré ; partisan d'un breton « moderne », très celtique mais taxé par beaucoup de « chimique » (pour lequel il a beaucoup suivi Goulven Pennaod); directeur de la revue d'idées Preder, nationaliste et intellectuelle, ainsi que de la revue plus politique et théorique Emsav dans les années soixante-dix ; auteur de poèmes assez cérébraux, influencés par la poésie allemande, comme il l'est par la philosophie allemande ; se consacre depuis l'échec de ses entreprises bretonnantes à la poésie en breton, mais aussi en français (devenu proche du Breton-Américain P. Keineg), récemment cité comme un des plus grands poètes bretonnants du temps par le critique américain W. Calin (1999).

8. Rieg Jestin, alias Raymond Gestin (Landerneau 1905-1991 Menton), spécialiste des Sumériens au Collège de France et à l'université d'Istanbul, depuis les années trente où il participa à des fouilles en Mésopotamie, et où il collabora à la revue Gwalarn, notamment par une étude philosophique retentissante alors, Bouddha hag ar Vouddhaegez (1936 « Bouddha et le Bouddhisme »), une première en langue bretonne. Ce fut également un artiste-peintre connu et reconnu qui a exposé à Paris, New York etc. Grand voyageur, il s'était fait par ailleurs connaître après la dernière guerre pour sés idées racistes et extrémistes, exprimées dans une feuilie qu'il dirigeait, An Nerzh (« La Force »), publiée de 1968 à 1990.

9. Dazoue, «bretonnisation » de l'irlandais ancien Dagda (= bon Dieu), le premier des dieux du panthéon druidique, selon les textes mythologiques irlandais du haut moyen âge; en breton reconstitué, da «bon $»+z$ /doue « dieu $»$.

10. «Yuzev lous ", soit " Juif sale » en fait ; c'est un calque français ; " sale Breton... » se dirait normalement kozh... « Sale mao » (comme disaient les... Arabes aux fayots bretons) rest rendu chez les auteurs bretonnants par kozh mao...

11. "kudenn ar vono- pe ar boligenezenn", mono- \& poly- + (?) genèse, ou mono-/polythéisme (?) néologismes. 
12. Touseg, pluriel tousegi « crapauds "; concernant l'origine de ce thème, on peut penser à un poème de J. Riou ainsi appelé, publié par Gwalarn en 1928 (mais il s'agit d'une poésie intimiste et personnelle, basée sur « la musique du I » et qui n'a rien à voir avec ces idées). Ce nom était donné, croit-on, dans des cercles restreints influencés par Ker Vreiz et chez les nationalistes intransigeants, dans les années 19601970 à des « régionalistes » comme. P.J. Hélias ou autres écrivains bretonnants issus de la mouvance de la Résistance, que séparait d'abord des « nationalistes » la querelle de l'orthographe (unifiée en 1941, puis concurrencée par l'《 universitaire » dans les années 50); ce terme injurieux et méprisant (dans le sens de « collabo », mais pris à l'envers) a été subverti dans un poème de mon collègue le Prof. Piriou, dans les années 1980, vu de la mouvance opposée, dite " gauchè et Bretagne» (qui signa parfois An Touseg). Sinon, la grenouille (de mare) ran a une tradition bien différente : le chant Gousperou ar Raned est très connu du peuple et toujours chanté (repris par de nombreux chanteurs médiatisés commeY.F. Kemener etc.), et reconnu depuis le Barzaz Breiz (1838) de La Villemarqué, sous la forme Ar Rannou (" Les Séries »), pour contenir au moins des restes de la méthode d'apprentissage mnémotechnique que I'on prêtre aux druides ; mais c'est un sujet de controverse, sur lequel on a beaucoup disserté, objet d'un récent livre de J.J. Boidron, Gousperoù ar raned (Dastum, i 995$)$.

13. YOD-KERC'H ( Bouillie d'avoine » plat traditionnel, mais réputé aphrodisiaque en breton) est, au contraire, une revue étudiante de type underground, d'esprit clairement libertaire ou en tout cas gauchiste, tout en étant dans la tradition nationaliste de l'emsav, mais de contenu très contestataire, selon l'esprit post68, pour une « libération» (entre autres sexuelle, comme chez Ginsberg, Kerouac et als le sexe étant, avec la " piste »-alcool et drogue etc. -, la motifémique principale de ces pages ronéotées, contenant $\mathrm{BD}$, poèmes et textes etc., qui sont parfois excellents). Elle est parue sur une dizaine d'années (de 1973 à 1982), mais certaines œuvres littéraires, publiées alors ainsi, de façon anonyme et un peu sous le manteau, ont été rééditées depuis. 